\title{
List of reviewers engaged in the Special Issues on Biosignal Processing
}

The articles published in Biomed Tech 2006; 51: 41-94; 281-376 and Biomed Tech 2007; 52: 5-168 were intensively reviewed, each by at least two referees. The reviewers were:

Bauernschmitt, R.; Deutsches Herzzentrum München Bonnemeier, H.; Medizinische Universität zu Lübeck Brix, G.; Bundesamt für Strahlenschutz München Burger, M.; Universität Erlangen Canisius, S.; Universitätsklinikum Gießen und Marburg Cebrián, A.; Universidad Politecnica de Valencia Christova, L.; Bulgarian Academy of Sciences, Sofia Dambier, M.; Robert Bosch GmbH, Stuttgart

Daumer, M.; Sylvia Lawry Zentrum für Multiple Sklerose Forschung e.V. München Dickhaus, H.; Fachhochschule Heilbronn

Dössel, O.; Universität Karlsruhe

Eichler, M.; Universität Heidelberg

Fleischer, Chr.; Technische Universität Berlin

Hofer, H.; Universität der Bundeswehr München

Hoffmann, K.P.; Fraunhofer Institut für Biomedizinische Technik, St. Ingbert

Hoppe, U.; Universität Erlangen-Nürnberg

Horsch, A.; Technische Universität München

Hoyer, D.; Universität Jena

Jordan, D.; Technische Universität München

Jordan, J.; Universitätsmedizin Berlin Charité

Kalthoff, O.; Hochschule Heilbronn

Kantelhardt, J.; Martin-Luther-Universität Halle-Wittenberg

Keller, P.; Max-Planck-Institut für Kognitions- und Neurowissenschaften, Leipzig

Klose, U.; Universität Tübingen

Koch, H.; Physikalisch-Technische Bundesanstalt Berlin

Krüger, T.; Universität Freiburg

Kuhn, K.; Technische Universität München

Leppek, R.; TransMIT-Zentrum für angewandte radiologische Forschung (ZARF), Marburg

Link, A.; Physikalisch-Technische Bundesanstalt Berlin

Luecke, D.; Universität Duisburg-Essen

Maier, C.; Hochschule Heilbronn

Malberg, H.; Forschungszentrum Karlsruhe GmbH/Universität Karlsruhe

Meffert, B.; Humboldt-Universität zu Berlin

Mikut, R.; Forschungszentrum Karlsruhe $\mathrm{GmbH}$

Noack, F.; Technische Universität Dresden

Nolte, G.; Fraunhofer FIRST, Berlin

Olthoff, A.; Universität Göttingen

Orglmeister, R.; Technische Universität Berlin

Penzel, T.; Universitätsmedizin Berlin Charité

Porta, A.; Universita degli Studi di Milano

Rüdiger, H.; Technische Universität Dresden

Sander-Thömmes, T.; Physikalisch-Technische Bundesanstalt Berlin

Schablowski-Trautmann, M.; Universität Heidelberg

Schanze, T.; Zentrum für Neurowissenschaften der RWTH Aachen

Staude, G.; Universität der Bundeswehr München

Stein, P.K.; Washington University School of Medicine, USA

Stieglitz, T.; Universität Freiburg

Stockmanns, G.; Universität Duisburg-Essen

Suhrbier, A.; Forschungszentrum Karlsruhe $\mathrm{GmbH}$

Trahms, L.; Physikalisch-Technische Bundesanstalt Berlin

Ungureanu, M.; Politehnica University of Bucharest, Romania 
Van Leeuwen, P.; Private Universität Witten-Herdecke Voss, A.; Fachhochschule Jena Weibezahl, K.F.; Forschungszentrum Karlsruhe GmbH

Wessel, N.; Universität Potsdam

Winterhalder, M.; Universität Freiburg

Witte, H.; Universität Jena

Wolf, W.; Universität der Bundeswehr München

Ziehe, A.; Fraunhofer-Institut für Rechnerarchitektur und Softwaretechnik Berlin 
Offenlegung der Inhaber und Beteiligungsverhältnisse gem. § 7a Abs. 1 Ziff. 1, Abs. 2 Ziff. 3 des Berliner Pressegesetzes: Gisela Cram, Rentnerin, Berlin; Dr. Annette Lubasch, Ärztin, Berlin; Elsbeth Cram, Pensionärin, Rosengarten-Alvesen; Margret Cram, Studienrätin i. R., Berlin; Verena Graß, Schülerin, Leimen; Brigitta Duvenbeck, Oberstudienrätin, Bad Homburg; Dr. Georg-Martin Cram, Unternehmens-Systemberater, Stadtbergen; Jens Cram, Student, Freiburg; Maike Cram, Stadtbergen; Renate Tran, Zürich; Gudula Gädeke M.A., Atemtherapeutin/Lehrerin, Tübingen; John-Walter Siebert, Pfarrer, Walheim; Dr. Christa Schütz, Ärztin, Mannheim; Dorothee Seils, Apothekerin, Stuttgart; Gabriele Seils, Journalistin, Berlin; Ingrid Cram, Betriebsleiterin, Tuxpan / Michoacan (Mexiko); Sabina Cram, Mexico DF (Mexiko); Prof. Dr. h.c. mult. Klaus G. Saur, Geschäftsführender Gesellschafter, München; Dr. Clara-Eugenie Seils, Oberstudienrätin i.R., Reppenstedt; Christoph Seils, Journalist, Berlin; Angelika Crisolli, kaufm. Angestellte, Hohenstein; Susanne Cram-Gomez, Mexico DF (Mexiko); Kurt Cram, Großhändler, Cancun (Mexiko); Silke Cram, Wissenschaftlerin, Mexico DF (Mexiko); Walter de Gruyter Stiftung, Berlin. 